\title{
Ultrashort Ultraviolet Free-Electron Lasers ${ }^{1}$
}

\author{
D. UMSTADTER \\ Center for Ultrafast Optical Science, University of Michigan, Ann Arbor, Michigan 48109-2099
}

AND

L. H. YU, E. JOHNSON, AND D. LI

National Synchrotron Light Source, Brookhaven National Laboratory, Upton, New York 11973

Received December 27, 1993; revised May 2, 1994

\begin{abstract}
In this work we combine elements of chirped pulse amplification (CPA) techniques, now familiar in solid-state lasers, with an amplifier based upon a seeded free-electron laser (FEL). The resulting device would produce amplified pulses of unprecedented brevity at wavelengths shorter than can be currently obtained by any tunable laser system. We use a subharmonically seeded FEL to illustrate the concept. Radiation from a Ti:sapphire laser is frequency-tripled and stretched optically to provide a coherent seed pulse for the FEL. When coupled to an electron beam inside a magnetic wiggler, the seed radiation introduces an additional energy modulation on the electron bunch, which has been prepared with an energy chirp to match the chirp in the optical pulse. The energy modulated electrons are then spatially bunched in a dispersion magnet and introduced to a wiggler configured to be resonant to a harmonic of the seed laser, providing additional frequency multiplication. The coherent radiation produced by these electrons is amplified as it traverses the wiggler and recompressed optically. The preservation of phase coherence provided by this scheme results in a device which can yield 4-fs pulses with $0.3 \mathrm{~mJ}$ at a central wavelength of ca. $88 \mathrm{~nm}$, easily the shortest duration amplified pulses produced by any laser. In this paper, we discuss various aspects of the concept, including the generation of short pulses, temporal stretching and compression, and potential applications of the device. The phase distortion during the wide bandwidth FEL amplification is discussed in detail, and is shown to be within the bounds required to produce a 4-fs pulse upon compression. (1) 1994 Academic Press, Inc.
\end{abstract}

\section{INTRODUCTION}

The quest for sources of radiation which produce pulses on the order of femtoseconds cuts across many disciplines of fundamental science, which can be attributed to the fact that many electronic processes occur on this time scale. An instrument capable of producing ultrashort pulses could be used to probe electron transfer reaction dy-

\footnotetext{
${ }^{1}$ Work performed under the auspices of the U.S. Department of Energy, under Contract DE-AC02$76 \mathrm{CH00016}$, The National Science Foundation under Contract PHY8920108, and the Office of Naval Research under Contract N00014-91-K-2005.
} 
namics in molecular and atomic systems, providing information about the most basic reaction mechanisms in chemistry, biology, and solid state physics. Employed in a pump-probe experiment, a laser of this type could provide "snap shots" of reactive systems where nuclear motion is important, since at the femtosecond level, molecular vibrations of even highly excited systems are essentially frozen.

One difficulty often encountered in such studies is the fact that many of the interesting systems are effectively dilute, either by their nature (for example, gas-phase systems, or transient radical species), or in the large number of quantum states they possess, which include multiphoton processes and molecular systems of even modest complexity. To address this problem, the candidate radiation source must be intense. It must also be noted that for many types of experiments, the radiation source must have wavelength tuning capability, since many phenomena have cross sections which depend strongly on the incident photon energy. Taken separately, each of these constraints provides a significant challenge to laser technology; collectively they have until recently represented an "insurmountable opportunity" which has only recently begun to yield to innovative approaches.

Foremost among these enabling technologies has been the development of chirped pulse amplification (CPA) techniques in solid state lasers (1). Utilizing these techniques, it is now possible to build compact lasers that produce both ultrashort pulses $(\tau \leqslant 50$ fs) and intensities as high as $10^{18} \mathrm{~W} / \mathrm{cm}^{2}$, several orders of magnitude higher than achieved even as recently as 5 years ago (2). For these extremely short pulses, transformlimited bandwidth is an important experimental consideration, since the minimum pulse width can be expressed as (3):

$$
\tau \simeq \frac{0.5}{\Delta \nu}=\frac{\lambda^{2}}{2 c \Delta \lambda}
$$

Thus shorter pulses may be produced by increasing bandwidth $(\Delta \lambda / \lambda)$ or reducing the wavelength. Shorter wavelength pulses have been generated by harmonic generation in crystals $(190 \mathrm{~nm})(4)$ or atomic vapors $(13 \mathrm{~nm})(5)$ but, as a result of their low power levels (nJ), they are of limited utility without further amplification. Shorter wavelength radiation than is currently available from laser sources is also attractive from the standpoint of the electronic transitions which become accessible.

For example, molecules such as $\mathrm{CH}_{4}, \mathrm{H}_{2} \mathrm{O}, \mathrm{CO}_{2}$, and most of the chlorofluorohydrocarbons simply do not absorb light at wavelengths longer than $200 \mathrm{~nm}$; all are species of tremendous importance in atmospheric photochemistry. DNA and other molecular targets in living cells are also known to be sensitive to radiation at wavelengths below $200 \mathrm{~nm}$, where specific absorption and consequent photochemical changes in chromophores within the molecule are thought to be responsible for the UV radiation damage observed in biological systems. This wavelength range is essentially inaccessible to current solid state laser systems, since the materials used in conventional laser amplifiers have a short wavelength cut-off near $\lambda \leqslant 180 \mathrm{~nm}$.

Concurrent with the development of CPA in solid-state lasers have been significant advances in accelerator technology. The performance of high brightness photocathode electron guns has recently routinely surpassed the threshold level required for practical 
single-pass free-electron lasers (FEL). Improvements have been made in methods to transport and compress the electron bunches to generate extremely high peak currents while maintaining the high brightness beams these guns can produce. Collectively, these developments have in turn led to a number of innovative proposals for constructing short wavelength FELs (6). One promising approach pursued at Brookhaven National Laboratory is that of the subharmonically seeded FEL $(7,8)$.

Briefly, the concept involves coupling the coherent radiation provided by a "conventional" pulsed laser with a high current electron bunch inside a magnetic wiggler designed to be resonant at the seed laser wavelength. This process introduces an energy modulation in the electron beam which is converted to a spatial modulation by a dispersive magnet. The microbunched electron beam is then transported to a second wiggler designed to be resonant to a harmonic of the original seed laser. The coherent radiation produced by these electrons is amplified as it traverses the wiggler. This approach has several significant advantages over oscillator configurations or singlepass designs which rely on "start-up from noise" or so-called self-amplified spontaneous emission (SASE) schemes.

In the seeded beam approach, the initial energy modulation is the result of interaction with a coherent optical field. As a consequence, the radiation produced in the FEL has the coherence properties and relative bandwidth of the seed laser. Compared to SASE devices, the external field induced energy modulation in the seeded beam approach has the further advantage of significantly reducing the length of the energy modulation wiggler. The concept of frequency multiplication in the electron beam is equally applicable to either approach, essentially treating the beam as a high quality nonlinear gain medium. As such, it has a distinct advantage over solid-state amplifiers in as much as it has a short wavelength cut-off determined primarily by the electron beam emittance. At the current state of the art, this places the FEL short wavelength limit below $75 \mathrm{~nm}(7)$, while improvements which appear to be technologically feasible have been proposed for an SASE device that would operate at $4 \mathrm{~nm}$ and below (9).

In this paper, we consider various aspects of a design that combines the attributes of the subharmonically seeded FEL, with the potential for short pulse generation afforded by chirped pulse compression techniques. We turn first to a discussion of CPA in solid-state lasers, as it forms the basis for the seed laser, and then turn our attention to the properties of the FEL required to exploit CPA to produce ultrashort pulses of UV radiation.

\section{CHIRPED PULSE AMPLIFICATION (CPA) IN SOLID-STATE LASERS}

CPA allows solid-state lasers to achieve much higher peak power than had been previously attained by direct amplification. In the CPA technique, the stored energy from a short pulse oscillator is extracted and stretched prior to amplification. By stretching the pulse, the input fluence to the amplifier remains constant while the intensity is reduced, which mitigates the problem of phase distortion in the amplification medium. The amplified pulses are then compressed to produce an extremely high peak power, short pulse. Stretching and compressing are achieved by utilizing gratings to disperse the frequencies of the pulse in such a manner that their path lengths prior to entering the amplifier differ. 
An essential condition for the amplification of a short pulse is that the bandwidth of the laser system be broad enough to accommodate the spectrum of frequencies from which it is comprised. The relationship between the pulse duration $\tau$ and bandwidth $\Delta \nu$ is given by the Fourier transform limit $\Delta \nu \tau \sim 0.5$. However, the amplifier medium generally has a wavelength dependent gain which results in a reduction of the pulse bandwidth (gain narrowing) and consequent increase in output pulse duration. The finite bandwidth of the other optical elements in the system may also limit the minimum pulse duration.

To date, the minimum amplified pulse length is $50 \mathrm{fs}$, achieved by CPA in a solidstate laser (10). In this case, the net bandwidth is $\Delta \lambda / \lambda=25 \mathrm{~nm} / 790 \mathrm{~nm}$ or $2.0 \%$. The titanium sapphire (Ti:sapphire) utilized in the amplifier has an extraordinarily large gain bandwidth $\left((\Delta \lambda)_{\mathrm{g}} \sim 2000 \AA\right)$ centered at $8000 \AA$ and extending up to $1 \mu \mathrm{m}$, corresponding to $\Delta \lambda / \lambda=25 \%$. Thus much shorter pulses are in principle possible, with the current limitation imposed by gain narrowing. New designs are expected to limit the total bandwidth to only $\Delta \lambda / \lambda=9 \%(\tau=15 \mathrm{fs}$ ) with an amplified energy of $10 \mathrm{~mJ}(11)$. Frequency-tripling this pulse in order to provide the seed pulse for injection into the FEL amplifier should actually improve the intensity contrast between the peak and the wings. However, walk-off of the beam for short pulses may limit the bandwidth of the harmonic. We conservatively estimate that the 264-nm seed pulse will have an energy of $20 \mu \mathrm{J}$ (assuming a conversion efficiency of only $0.2 \%$ ) and a bandwidth of at least $\Delta \lambda / \lambda=4 \%$, which, by Eq. [1], corresponds to an 11-fs pulse containing 12 optical cycles. Asaki et al. (12) have already demonstrated frequency doubled pulses of 11 fs from a 15-fs fundamental using a $400-\mu \mathrm{m}$ BBO crystal. The fourth harmonic has also been produced with femtosecond fundamental pulses of slightly longer duration (4).

\section{APPLICATION OF CPA TO A SUBHARMONICALLY SEEDED FREE-ELECTRON LASER}

Without chirping the electron beam energy, a high gain FEL's gain bandwidth is approximately given by a Pierce parameter $\rho(13,14)$, which is roughly the growth rate per wiggler period divided by $4 \pi$. For an ultraviolet FEL, a Pierce parameter of $\rho \simeq 0.1 \%$ would be typical. Therefore, to amplify a chirped pulse with a few percent bandwidth, the energy of the electron pulse should be chirped to match the resonance condition,

$$
\lambda(t)=\frac{\lambda_{\mathrm{w}}}{2 \gamma^{2}(t)}\left(1+\mathrm{a}_{\mathrm{w}}^{2}\right)
$$

where $\lambda(t)$ is the laser wavelength, $\gamma(t)$ is the electron beam energy, $\lambda_{\mathrm{w}}$ is the wiggler period, and $\mathrm{a}_{\mathrm{w}}=e \lambda_{\mathrm{w}} B_{\mathrm{w}} / 2 \pi m c$ is known as the scaled wiggler vector potential with wiggler field $B_{\mathrm{w}}$ (rms value on axis). The time $t$ is used to denote the longitudinal position along the radiation and electron pulse.

The subject of energy chirped FELs has been previously explored in an oscillator configuration (15). However, to create a chirped output radiation pulse that can be compressed to a short pulse the order of femtoseconds duration, the pulse must be very accurately chirped; i.e., from the head to the tail of the pulse, the optical phase 
relationship should be as coherent as if it had been originally stretched from a femtosecond pulse. It is difficult to generate such coherence starting from noise, as occurs in oscillator or SASE FEL configurations. Hence, from the standpoint of phase coherence, the choice of a chirped pulse seeded single-pass FEL seems the most promising configuration to pursue. The subharmonically seeded beam approach developed for the Brookhaven National Laboratory deep ultraviolet free electron laser (DUV-FEL) $(7,8)$, provides a framework for the present discussion, with the overall scheme shown schematically in Fig. 1. For the FEL, pulses from the previously described solid state laser will be stretched to provide a 5-ps chirped pulse to seed the FEL.

In this case, the electron source is an RF photocathode gun providing up to $4 \mathrm{nC}$ of charge in a nominal 20-ps pulse. The electrons are accelerated to an energy of up to $310 \mathrm{MeV}$ by five SLAC-type linac sections. The last linac section is used to tune the electron beam energy, or alternatively to generate energy chirping to match the chirped input seed laser beam. By introducing the energy chirp right in front of the wiggler, the complexities associated with transport and emittance preservation of electron beams with a relatively large energy spread are eliminated. In our example, the electron beam has an energy centered at $267 \mathrm{MeV}$, a peak current of $256 \mathrm{~A}$, and a normalized rms emittance of $6.4 \pi \mathrm{mm}$ mrad.

This high brightness electron beam is transported to an initial 2-m-long wiggler, with period of $\lambda_{\mathrm{w} 1}=3.5 \mathrm{~cm}$ and maximum on-axis field of $0.76 \mathrm{~T}$, where it interacts with the seed laser radiation. This so-called "modulator" is designed to be resonant to $264 \mathrm{~nm}$, the wavelength of the seed radiation, and introduces a small energy modulation on the electron beam. The electron beam then enters a dispersion section 20 $\mathrm{cm}$ in length with a $0.32-\mathrm{T}$ magnetic field.

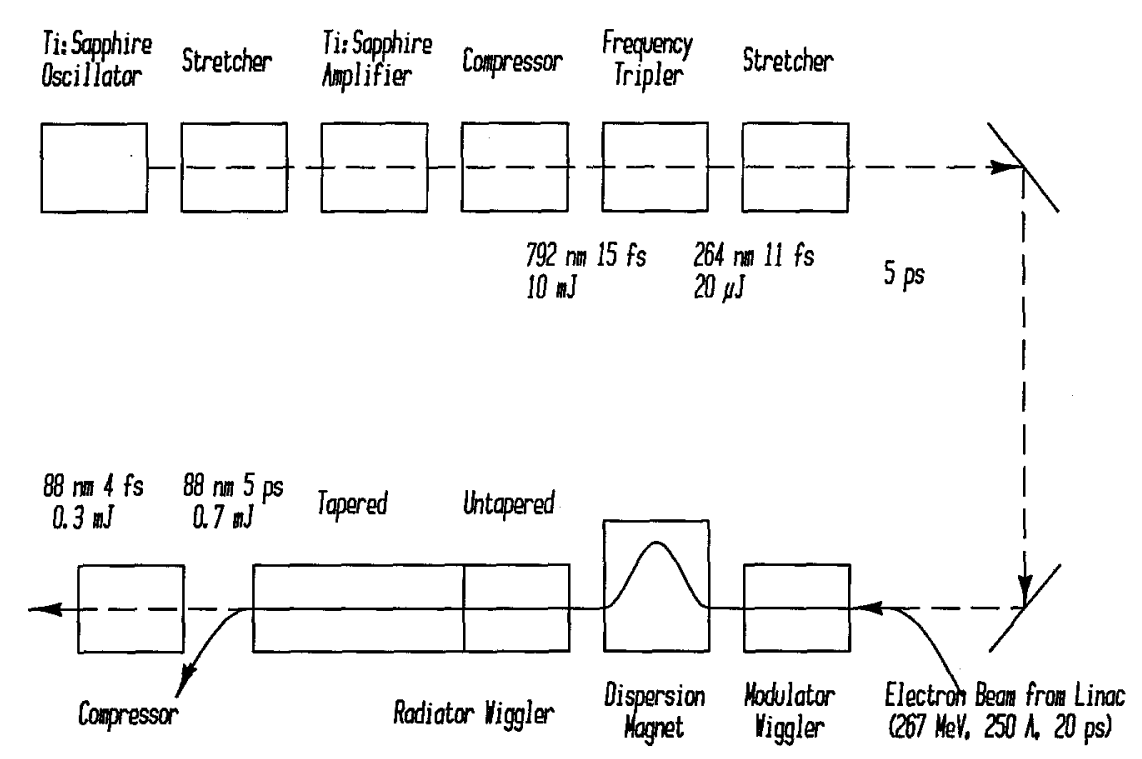

FIG. 1. A schematic diagram showing the experimental arrangement. 
The function of this magnet is to convert the energy modulation of the beam into a spatial bunching with a strong third harmonic component at $88 \mathrm{~nm}$. After it is microbunched, the beam enters a second wiggler, known as the "radiator," resonant for $88-\mathrm{nm}$ radiation having a maximum on-axis field of $0.75 \mathrm{~T}$ and $\lambda_{\mathrm{w} 2}=2.2 \mathrm{~cm}$. When the coherently bunched beam enters the second wiggler magnet, there is a rapid coherent generation of $88-\mathrm{nm}$ radiation within the first meter where the intensity of the radiation has a characteristic quadratic dependence on the distance traversed in the wiggler. There is then a transition to exponential growth which continues until 6 $\mathrm{m}$ into the wiggler where the $88-\mathrm{nm}$ radiation approaches saturation. At this point the magnetic field of the wiggler is adjusted or "tapered" to maintain resonance between the photon field and the electron beam. This technique allows additional energy extraction from the electron beam beyond that possible with a fixed field wiggler. Here we have used $264-\mathrm{nm}$ seed as an example to obtain $88-\mathrm{nm}$ output radiation. The system is actually tunable over a wide wavelength range determined by the chirped pulse laser used to generate the seed radiation.

We now turn to the key issue in the system: the preservation of the phase coherence of the seed pulse in the FEL. According to the theory of chirped pulse amplification (16), the phase distortion should be maintained below $\pi$, so that after compression the different frequency components of the chirped pulse will be coherently superimposed together at the center of the pulse.

The phase and amplitude of the output radiation depend on the current and energy detuning of the electron beam and, within a bunch, both the current and energy are functions of time. Although the energy of the electron beam is linearly chirped to match the linear chirp of the seed laser pulse wakefields generated during the acceleration introduce an additional nonlinear chirping, which results in energy detuning. Further, because of its finite bandwidth, the input chirped laser pulse intensity is also a function of time. Each of these factors contributes to phase and amplitude distortion in the FEL. To minimize the collective impact of these effects we only use the central 5 -ps part of the electron bunch, where the current is near the maximum and varies only a few percent, and the energy detuning is also minimized. During this $5 \mathrm{ps}$, the seed laser wavelength $\lambda$ is chirped through a $4 \%$ bandwidth. To resonantly match the seed laser, the electron beam energy $\lambda$ is chirped through $2 \%$. These parameters form the basis of our calculations.

The electron distribution (current as a function of time within the pulse) is based on the BNL DUV-FEL design operating in a configuration that provides a 20-ps-long pulse with peak current of $256 \mathrm{~A}$ and a relatively flat top, suitable for chirped pulse amplification. The relevant operating parameters are provided in Table I. To include wakefield effects, we assumed an energy chirp of $0.8 \%$ in 20 ps with cubic time dependence in addition to the applied linear chirping. The local energy spread (the energy spread within a slice of the electron pulse thin enough so that the energy chirping within the slice is negligible) is less than $0.1 \%$ FWHM. The laser pulse is assumed to be gaussian and linearly chirped through $4 \%$ in the central 5 ps with a FWHM bandwidth of $2 \%$, and a peak power of $4.6 \mathrm{MW}$. For the FEL simulation, we used a modified TDA code to calculate the output phase and amplitude $(17,8)$. For a more detailed description of the simulation, we refer to Ref. (8). 
TABLE 1

DUV-FEL Specifications

\begin{tabular}{ll}
\hline RF photocathode gun current & $4 \mathrm{nC}$ \\
RF photocathode gun pulse width & $20 \mathrm{ps}$ \\
Linac energy & $310 \mathrm{MeV}$ \\
Linac peak current & $256 \mathrm{~A}$ \\
Linac rms emittance & $6.4 \pi \mathrm{mm} \mathrm{mrad}$ \\
Modulator wiggler length & $2 \mathrm{~m}$ \\
Modulator wiggler period & $3.5 \mathrm{~cm}$ \\
Modulator wiggler magnetic field & $0.76 \mathrm{~T}$ \\
Dispersion section length & $20 \mathrm{~cm}$ \\
Dispersion section magnetic field & $0.32 \mathrm{~T}$ \\
Radiator wiggler period & $2.2 \mathrm{~cm}$ \\
Radiator wiggler magnetic field & $0.75 \mathrm{~T}$ \\
\hline
\end{tabular}

For the calculation, we make an approximation related to the impact of slippage on ultrashort pulse amplification in an FEL. The slippage problem arises from the fact that the electron pulse moves more slowly than the laser pulse. If the laser pulse is much shorter than the slippage distance, the electrons interacting with the laser pulse at the beginning of the wiggler would slip away from the laser pulse before the end of the wiggler, and the FEL efficiency would be reduced significantly. The slippage in our example scales to roughly a 0.1-ps transit time difference for the electrons and photons. Within this $0.1 \mathrm{ps}$, the energy of the electrons that interact with the laser light changes due to chirping. However, $0.1 \mathrm{ps}$ is much smaller than either the laser pulse or electron bunch, so the variations of current and energy are negligible, and therefore are not included in our analysis. This is also related to the validity of using TDA code, which is a time-independent single frequency 3D code; in principle, we should use 4D code with full time dependence. However, the variation of wavelength within the slippage distance is also small, so the phase shift variation within the slippage should be negligible. If we divide the pulse into many slices with each slice length less than the slippage distance, we are justified in our use of 3D code to calculate the phase shift within each slice independently. A more rigorous calculation should compare a $1 \mathrm{D}$ chirped pulse calculation with a 2D time-dependent calculation using a simulation code such as GINGER (18).

To describe the phase relation between the input laser and output laser, we write the input field as

$$
A_{1}=a_{1}(t) \cos \left[k_{1} z-\omega_{1} t+\phi_{1}(t)\right],
$$

while the output field is

$$
A_{2}=a_{2}(t) \cos \left[k_{2} z-\omega_{2} t+\phi_{2}(t)\right]
$$

where $k_{1}, k_{2}, \omega_{1}, \omega_{2}$ are the wavenumber and frequency for the center of the chirped input and output pulses, respectively, for $\lambda_{1}=264 \mathrm{~nm}$ and $\lambda_{2}=88 \mathrm{~nm}$. The output 
phase distortion is then given by the nonlinear time-dependent part of the phase $\phi(t)$ $=\phi_{2}(t)-n \phi_{1}(t)$, where $n=\omega_{2} / \omega_{1}$ is the frequency multiplication ratio (in our example, $n=3$ ). Any term in $\phi(t)$ which is linear in $t$ only represents a frequency shift.

The phase difference $\phi$ between $\phi_{1}$ and $\phi_{2}$ is determined by the complicated FEL interaction and the electron microbunching process in the dispersion section through the electron phase shift. It is convenient to write $\phi$ as a sum of two terms: $\phi=\phi_{\text {disp }}$ $+\phi_{\text {fel }}$. The first term $\phi_{\text {disp }}$ is determined by the electron phase shift in the dispersion section. We have

$$
\phi_{\mathrm{disp}}=\frac{d \psi}{d \gamma}\left(\overline{\gamma(t)}-\gamma_{0}\right)+\phi_{0}
$$

where $d \psi / d \gamma$ is the dispersion strength defined in Ref. (8), which is the electron phase shift per unit energy deviation from a reference energy $\gamma_{0}$ in the dispersion section. If the $j$ th electron passes the dispersion section delayed by $d t_{j}$ because its energy is less than the reference energy $\gamma_{0}$ by $d \gamma$, then the phase delay is $d \psi_{j}=\omega_{2} d t_{j}$ (in our example $d \psi / d \gamma=3) . \overline{\gamma(t)}$ is the average electron energy at time $t$, and $\phi_{0}$ is a constant determined by the distances between the dispersion section and the wigglers. It is easily seen that a linear chirping in energy $(d \gamma / d t) t$ introduces a frequency shift $\delta \omega=(d \psi / d \gamma)(d \gamma / d t)$, while a nonlinear chirping (nonlinear energy time dependence generated, e.g., by wakefield) introduces a phase distortion. The second term $\phi_{\mathrm{fel}}$ is the phase shift referenced to the phase of the electrons with the average energy $\overline{\gamma(t)}$, and is a function of current, energy detuning, and the input laser intensity. The output power and $\phi_{\mathrm{fel}}$ on the axis are calculated for each individual slice of the electron pulse using the modified TDA code, providing the results shown in Fig. 2 and Fig. 3. A calculation of $\phi_{\mathrm{fel}}$ off axis by an rms electron beam radius yields similar results. It is interesting

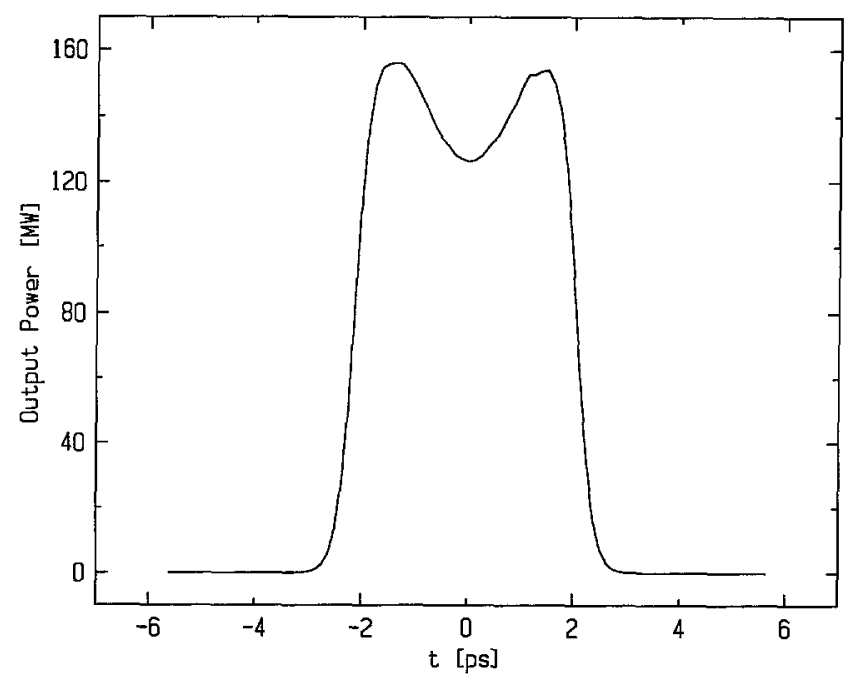

FIG. 2. Output power as a function of time. 


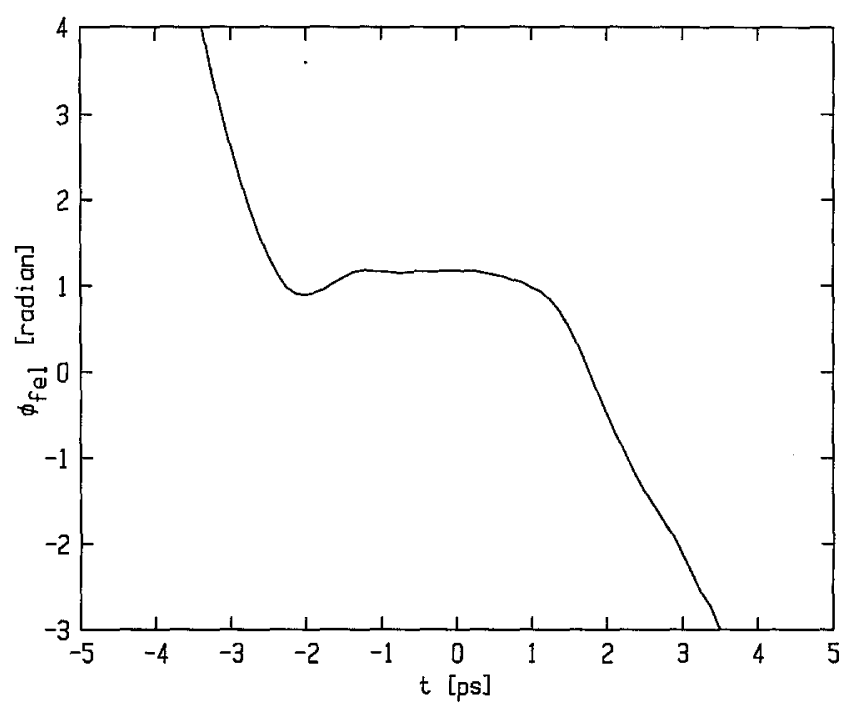

FIG. 3. Phase shift as a function of time.

to note that the output power has a dip at the center of the pulse. The optimum input laser pulse peak intensity should be $2 \mathrm{MW}$. However, for our calculation, we actually set the peak seed laser intensity to $4.6 \mathrm{MW}$ to increase the output pulse length and bandwidth, which gives rise to the dip.

Our calculations show that under these conditions, the 4-ps center slice provides most of the FEL gain while maintaining the intensity variation below $25 \%$, phase distortion (the nonlinear part of $\phi_{\mathrm{fel}}$ ) within $0.6 \mathrm{rad}$, with less than $0.2 \mathrm{rad}$ of nonlinear phase distortion introduced by $\phi_{\text {disp }}$. As previously mentioned, the seed laser pulse wavelength is chirped through $4 \%$ bandwidth within the central $5 \mathrm{ps}$, and the chirping rate is $4 \% / 5 \mathrm{ps}=0.8 \% / \mathrm{ps}$, hence the central 4-ps output radiation chirped through $0.8 \times 4=3.2 \% \approx 3 \%$ of bandwidth. This means that the output radiation has a $3 \%$ bandwidth, and can be compressed to $5 \mathrm{fs}$, as shown by Eq. [1]. The chirped pulse has a $150-\mathrm{MW}$ peak power and 4-ps pulse length with a pulse energy of about $0.6 \mathrm{~mJ}$, so when it is compressed to $5 \mathrm{fs}$, the resulting peak power approaches $60 \mathrm{GW}$, even if the compressor has a $50 \%$ loss.

This requires compression of a factor of 1000 , which is achievable by current standards although to operate at short wavelengths, the compressor system will consist of figured mirrors and flat gratings, since transmission optics are unavailable at these wavelengths. By use of all-reflective optics, the stretcher-compressor pair will be matched for all orders of group velocity dispersion, and coatings optimized for high reflectivity at $88 \mathrm{~nm}$ should yield a device with much better efficiency than the $50 \%$ loss figure used in this discussion.

If the seed laser bandwidth were improved to $4 \%$ at $264 \mathrm{~nm}$, with other conditions the same, the required input peak power would be reduced to about $2.3 \mathrm{MW}$, and the output bandwidth would be $4 \%$. The resultant pulse energy would be $0.7 \mathrm{~mJ}$, and could be compressed to $4 \mathrm{fs}$ with a peak power of $100 \mathrm{GW}$, even assuming a $50 \%$ loss for the compressor. 


\section{CONCLUSIONS}

The final peak output power provided by this scheme will be the order of $100 \mathrm{GW}$ at a repetition rate of $10 \mathrm{~Hz}$. It should be mentioned that short wavelengths can also be efficiently generated by high-order harmonic generation in a nonlinear medium. Although the $88 \mathrm{~nm}$ pulse could be produced by harmonic generation of the fundamental wavelength in an atomic vapor (the ninth harmonic), the pulse would not be as short and the power would be at least eight orders of magnitude lower. However, optimization of phase matching for greater efficiency can be accomplished by frequency mixing (20) the 790-nm seed pulse with the amplified 88-nm pulse. In this case, pulses could be produced with soft $\mathrm{x}$-ray wavelengths similar to, but with much shorter pulsewidths than, those that might be obtained by several proposed schemes to use Compton backscattering of light from an energetic electron beam (2l). Shorter pulse durations and shorter wavelengths may also be produced with this FEL design using a higher harmonic, albeit with a reduction in peak power. The peak power vs wavelength

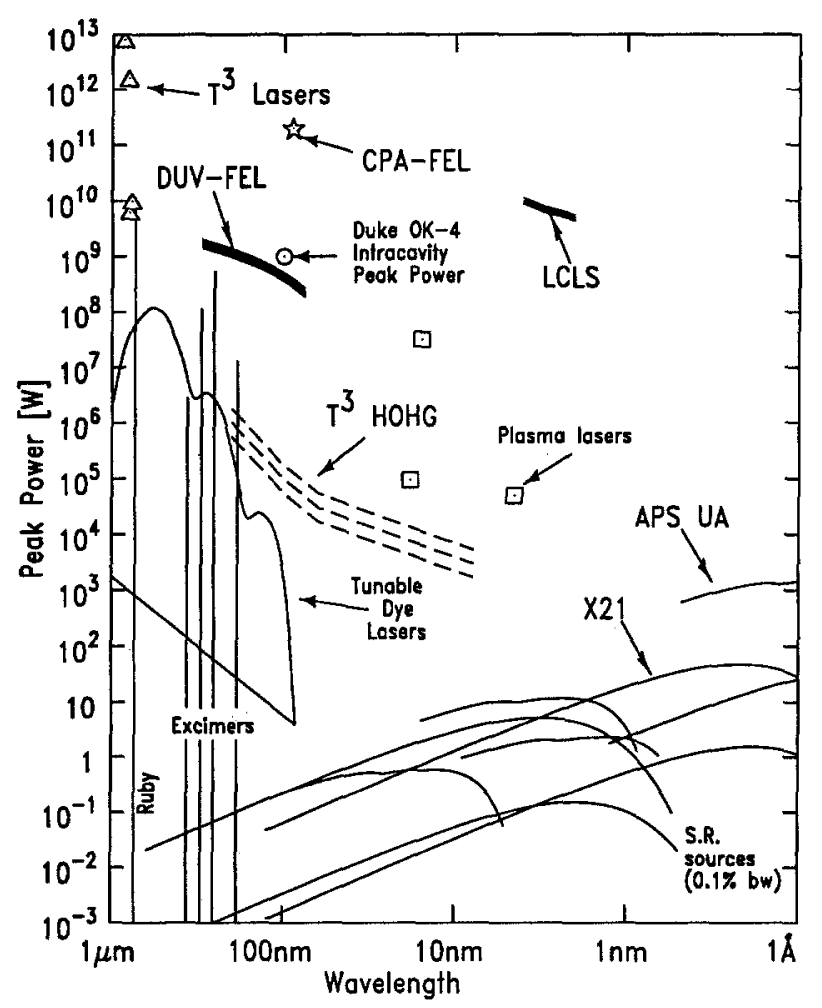

FIG. 4. Comparison of CPA-FEL with other sources. Acronyms: $T^{3}$ (table-top terawatt, solid-state laser); CPA-FEL (chirped pulse amplification free-electron laser, as proposed in this paper); DUV-FEL (deep ultraviolet free-electron laser); $T^{3} \mathrm{HOHG}$ (table-top terawatt laser high-order harmonic generation, in an atomic vapor); LCLS (linear coherent light source, amplified spontaneous emission from $60-\mathrm{m}$ wiggler on SLAC); APS UA (advanced photon source undulator A); X21 (hybrid wiggler on Nat. Sync. Light Source Ring); Duke OK-4 (1-GeV storage ring with Novosibirsk optical klystron, intracavity power in giant pulse mode); S.R. sources (synchrotron radiation sources, into $0.1 \%$ b.w.). 
of the CPA-FEL configuration described above is compared with other existing and proposed source technologies in Fig. 4.

Many important technical issues remain which require further study. For example, the effect of the jitter of the electron bunch relative to the RF phase of the accelerator is to cause an electron energy jitter due to the electron energy chirping. If this jitter becomes comparable to the FEL gain bandwidth, the output power of the FEL will be reduced. The detuning bandwidth of our FEL example is about $0.2 \%$. Since the electron energy is chirped $2 \%$ within 5 ps, a $0.2 \%$ change in energy occurs in 0.5 ps. This sets the tolerance of pulse timing jitter to about $0.5 \mathrm{ps}$, which is within the state of art for a laser-triggered photocathode, although a more quantitative analysis of this effect is still needed.

On the basis of this preliminary analysis, it appears to be entirely technically feasible to utilize CPA techniques with an FEL-based harmonic generator and amplifier to produce radiation of unprecedented brevity and peak power at wavelengths significantly shorter than are currently available from "conventional" CPA technology. If we can increase the seed laser bandwidth, increase the energy chirping by utilizing a higher frequency accelerator cavity in front of the wiggler, and improve the flatness of the center of the electron pulse, we can further increase the output bandwidth and shorten the pulse. We hope that this work will stimulate interest within the scientific community, so that they may consider the advances such a source might portend for their own work.

\section{ACKNOWLEDGMENTS}

We would like to thank J. Gallardo for providing the electron beam parameters of the BNL-ATF for the calculations, and S. Krinsky, I. Ben-Zvi, L. DiMauro, D. McWhan, G. Mourou, G. Korn, and E. Esarey for their useful comments and suggestions.

\section{REFERENCES}

1. P. Maine, D. Strickland, P. Bado, M. Pessot and G. Mourou, IEEE J. Quantum Electron. QE24, 398 (1988).

2. G. Mourou and D. UMSTadter, Phys. Fluids B 4, 2315 (1992).

3. M. Born AND E. Wolf, "Principles of Optics," p. 486, Pergamon, New York, 1970.

4. J. Ringling, O. KitTelmann, F. NOACK, G. KorN, AND J. SQuier, Opt. Lett. 18, 2035 (1993).

5. X. F. Li, A. L'Huillier, M. Ferray, L. A. Lompre, and G. Mainfray, Phys. Rev. A 39, 5751 (1989); J. F. Ward and G. H. C. New, Phys. Rev, 185, 57 (1969); A. MCPherson, G. Gibson, H. Jara, U. Johann, T. S. Luk, I. A. MCINTYRE, K. Boyer, AND C. C. Rhodes, J. Opt. Soc. Am. B 4, 595 (1987); K. C. Kulander and B. W. Shore, Phys. Rev. Lett. 62, 524 (1989); M. Ferray, A. L'Huillier, X. F. Li, L. A. Lompré, G. Mainfray, and C. Manus, J. Phys. B 21, L31 (1988); X. F. Li, A. L'Huillier, M. Ferray, L. A. Lompré, and G. Mainfray, Phys. Rev. A. 39, 5751 (1989); J. H. Eberly, Q. Su, and J. Javanainen, Phys. Rev. Lett. 62, 881 (1989).

6. I. BEN-ZVI AND H. WINICK (Eds.), Towards Short Wavelength FEL Workshop, held May 21-22, 1993 at Brookhaven National Laboratory, BNL-49651.

7. S. KRINSKY (Ed.), "Brookhaven National Laboratory DUV Free Electron Laser Conceptual Design Report," December 1993, BNL-49713.

8. L. H. YU, Phys. Rev. A 44, 5178 (1991).

9. M. CORNACCHIA AND H. WinICK (Eds.), Workshop on Fourth Generation Light Sources, Held 24-27 February, 1992 at the Stanford Synchrotron Radiation Laboratory, SSRL publication 92A2. 
10. J. V. Rudd, G. Korn, S. Kane, J, SQuier, G. Mourou, And P. Bado, Opt. Lett. 18, 2044 (1993).

11. C. BARTY, private communication.

12. M. T. Asaki, C.-P. Huang, D. Garvey, J. Zhou, M. Murnane, and H. C. Kapteyn, "Proc. of SPIE, Ultrafast Pulse Generation and Spectroscopy," Vol. 1861, p. 37, SPIE, Bellingham, WA, 1993.

13. R. Bonifacio, C. Pellegrini, ANd L. M. Narducci, Opt. Commun. 50, 373 (1984).

14. S. KRINSKY AND L. H. YU, Phys. Rev. A 35, 3406 (1987).

15. G. T. MOORE, Nucl. Instrum. Methods Phys. Res. A272, 302 (1988).

16. A. E. SieGMAN, "Lasers," Chap. 10, p. 386, University Science Books, 1986.

17. T. M. Tran and J. S. WUrTele, Comput. Phys. Commun. 54, 263 (1989).

18. W. M. Fawley, A. M. Sessler, and E. T. SCharlemann, in "Proceedings of the Particle Accelerator Conference, Washington, 1993," Lawrence Berkeley Laboratory Report LBL-33285, to appear.

19. This figure was developed from the following sources: K.-J. KIM AND A. SEsSLER, Science 60, 88 (1990); S. SUCKeWER AND C. H. SkInNER, Science 247, 1553 (1990); L. B. DaSilva, B. J. MacGowan, S. Mrowka, J. A. Kowch, R. A. London, D. L. Mathews, AND J. H. Underwood, Opt. Lett. 18, 1174 (1993); Table top terawatt $\left(T^{3}\right)$ systems and FEL projects from reference above; Synchrotron sources based on facility parameters calculated using code developed by S. L. HulberT AND J. M. WeBBER, Nucl. Instrum. Methods Phys. Res. A319, 25 (1992).

20. P. L. SHKOLNiKov, A. E. KAPLAN, AND A. LAGO, Opt. Lett. 18, 1700 (1993).

21. A. Luccio, G. Matone, L. Miceli, AND G. Giordano, Laser and Particle Beams 8, 383 (1990). 